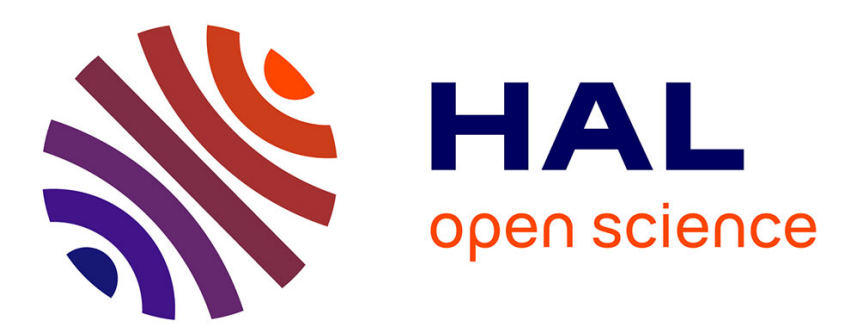

\title{
Efficient Equilibrated Flux Reconstruction in High Order Raviart -Thomas Space for Discontinuous Galerkin methods
}

\author{
Igor Mozolevski, Edson Luis Valmorbida
}

\section{To cite this version:}

Igor Mozolevski, Edson Luis Valmorbida. Efficient Equilibrated Flux Reconstruction in High Order Raviart -Thomas Space for Discontinuous Galerkin methods. 2016. hal-01385637

\section{HAL Id: hal-01385637 \\ https://hal.science/hal-01385637}

Preprint submitted on 21 Oct 2016

HAL is a multi-disciplinary open access archive for the deposit and dissemination of scientific research documents, whether they are published or not. The documents may come from teaching and research institutions in France or abroad, or from public or private research centers.
L'archive ouverte pluridisciplinaire HAL, est destinée au dépôt et à la diffusion de documents scientifiques de niveau recherche, publiés ou non, émanant des établissements d'enseignement et de recherche français ou étrangers, des laboratoires publics ou privés. 


\title{
Efficient Equilibrated Flux Reconstruction in High Order Raviart -Thomas Space for Discontinuous Galerkin methods
}

Igor Mozolevski and Edson Luiz Valmorbida

\begin{abstract}
We develop an efficient and computationally cheap method of equilibrated fluxes reconstruction for high - order dG solutions to elliptic problems using a specific computational basis in high order Raviart - Thomas space. The computational basis is designed in such a way that coordinates of equilibrated fluxes with respect to this basis can be easy calculated from the moments of the numerical fluxes of dG method. Some applications of this method in implementation of a posteriori error estimators for elliptic boundary value problems are considered.
\end{abstract}

\section{Introduction}

Equilibrated fluxes reconstruction in Raviart -Thomas space is used in finite element methods for development of fully computable (not involving unknown constants), efficient and reliable a posteriori error error estimates for elliptic, convectiondiffusion and parabolic problems, see e.g [19, 13, 14, 7, 8, 4, 22, 15]. As an another important application the equilibrated velocity recuperation from a discontinuous Galerkin solution to the Darcy equation in the multiphase flow in heterogeneous porous media should be mentioned, see [12,11]. One of the attractive properties of the equilibrated fluxes a posteriori error error estimates is the robustness in respect to the order of polinomial approximation (cf. [4],[15]), whereas the efficiency of residual type estimates can decrease with the degree (cf. [20], [5]).

Owing to the local conservation properties, the discontinuous Galerkin (dG) finite element methods allow easy flux reconstruction in Raviart-Thomas space by locally prescription of the numerical flux moments as the degrees of freedom, cf.

Igor Mozolevski

Federal University of Santa Catarina, Brazil, e-mail: igor.mozolevski@ufsc.br

Edson Luiz Valmorbida

Federal University of Technology - Paraná, Brazil, e-mail: edsonvalmorbida@utfpr.edu.br 
[9]. Such an approach offers cheap and efficient computational algorithm for implementation of flux reconstruction in lowest order Raviart-Thomas space.

Nevertheless, reconstruction of equilibrated fluxes from the prescribed moments of discrete numerical fluxes in higher order Raviart-Thomas space can be computationally involved procedure. In this article we introduce a specific modal basis in high order Raviart-Thomas space such that calculation of reconstructed flux coefficients in respect to this from the prescribed moments is extremely easy owing to orthogonal properties of edge elements of the basis. Using this tool we develop an efficient and computationally cheap method of equilibrated fluxes reconstruction from high-order $\mathrm{dG}$ solutions to elliptic problems. To demonstrate the potential of the method we consider an application to adaptive mesh refinement, where the method is used for equilibrated fluxes calculation needed for a posteriori error estimator.

\section{Modal basis in high order Raviart-Thomas space}

Let $\Omega$ be a polygonal domain in $\mathbb{R}^{2}$. Let us denote by $H^{k}(\Omega)$ the Sobolev space of order $k \in \mathbb{N}_{0}$. The space of vector functions $\mathbf{u} \in\left[L^{2}(\Omega)\right]^{2}$ with weak divergence $\nabla \cdot \mathbf{u}$ in $L^{2}(\Omega)$ is denoted by $H(\operatorname{div}, \Omega)$. The reader is referred to [1] and [3] among others, where standard properties of the Sobolev and $H($ div $)$ spaces are exposed.

For discrete approximation of $H(\operatorname{div})$ spaces let us define in $\Omega$ a shape regular family $\mathscr{T}_{h}$ of triangular meshes (see e.g. [6]), where $h=\max _{T_{\in} \mathscr{T}_{h}} h(T)$ denotes the mesh size and $h(T)$ is the diameter of the mesh element $T$. We denote the set of all mesh edges as $\mathscr{E}$ and decompose it in the set $\mathscr{E}$ i of all interior edges (interfaces between adjacent mesh elements) and the set of all boundary faces $\mathscr{E} \partial$. Next we define vector field $\mathbf{n}_{\mathscr{E}}: \mathscr{E} \rightarrow \mathbb{R}^{2}$ of edge normals, where $\mathbf{n}_{\mathscr{E}}(E)=\mathbf{n}_{E}$ is the fixed unit vector orthogonal to $E$ which coincides with the external normal to $\partial \Omega$ on the boundary edges. We also denote as $\mathbf{n}_{T}$ the external normal to $\partial T$ for any mesh element $T$. For $E \in \mathscr{E}$ we denote by $T_{E}=\left\{T \in \mathscr{T}_{h}: E \subset \partial T\right\}$ the set of all mesh elements sharing the edge $E$.

For any triangle $T \in \mathscr{T}_{h}$ the local Raviart-Thomas space is defined by

$$
\mathbb{R} \mathbb{T}^{k}(T)=\left[\mathbb{P}_{k}(T)\right]^{2}+\left(\begin{array}{l}
x \\
y
\end{array}\right) \mathbb{P}_{k}(T),
$$

where $\mathbb{P}_{k}(T)$ denotes the space of polynomials in $T$ of degree less on or equal to $k \in \mathbb{N}_{0}$. For $\mathbf{u} \in \mathbb{R} \mathbb{T}^{k}(T)$ the degrees of freedom are given by

$$
\begin{gathered}
\int_{\partial T}\left(\mathbf{u} \cdot \mathbf{n}_{T}\right) p, \quad \forall p \in \mathbb{P}_{k}(\partial T) ; \\
\int_{T} \mathbf{u} \cdot \mathbf{q}, \quad \forall \mathbf{q} \in\left[\mathbb{P}_{k-1}(T)\right]^{2} \quad \text { if } k \geq 1 .
\end{gathered}
$$


Associated with the triangulation $\mathscr{T}_{h}$ the global Raviart-Thomas finite element space is defined as

$$
\mathbb{R} \mathbb{T}^{k}\left(\mathscr{T}_{h}\right)=\left\{\mathbf{u}_{h} \in H(\operatorname{div}, \Omega)\left|\mathbf{u}_{h}\right|_{T} \in \mathbb{R}^{k}(T), \forall T \in \mathscr{T}_{h}\right\} .
$$

The computational implementation of $\mathbb{R} \mathbb{T}^{0}$ elements is typically included in finite element software packages and it was carefully discussed in [2]. Here we aim at introducing a computational basis in high order $\mathbb{R}^{k}\left(\mathscr{T}_{h}\right)$ such that the coordinates of an element in this basis can be easily calculated from its degrees of freedom (2) - (3). We start with the definition of the basis in the master element and then extend the definition to any $T \in \mathscr{T}_{h}$ using Piola transformation.

Let us consider the reference triangle $\hat{T}$ with vertexes

$$
\begin{gathered}
\hat{\mathbf{v}}_{1}=(-1,-1)^{\prime}, \hat{\mathbf{v}}_{2}=(1,-1)^{\prime}, \hat{\mathbf{v}}_{3}=(-1,1)^{\prime} ; \\
\hat{T}=\left\{\hat{\mathbf{v}}^{1}, \hat{\mathbf{v}}^{2}, \hat{\mathbf{v}}^{3}\right\}=\left\{(r, s)^{\prime} \mid r, s \geq-1 ; r+s \leq 0\right\} .
\end{gathered}
$$

For any $T \in \mathscr{T}_{h}, T=\left\{\mathbf{v}^{1}, \mathbf{v}^{2}, \mathbf{v}^{3}\right\}, \quad \mathbf{v}^{1}=\left(x_{1}, y_{1}\right)^{\prime}, \mathbf{v}^{2}=\left(x_{2}, y_{2}\right)^{\prime}, \mathbf{v}^{3}=\left(x_{3}, y_{3}\right)^{\prime}$ we fix the canonical affine application $\pi_{T}: \hat{T} \rightarrow T$ as:

$$
\pi_{T}(r, s)=-\frac{r+s}{2} \mathbf{v}^{1}+\frac{r+1}{2} \mathbf{v}^{2}+\frac{s+1}{2} \mathbf{v}^{3}=(x(r, s), y(r, s))^{\prime} .
$$

The Piola transformation corresponding to $\pi_{T}$ is defined for $\hat{\mathbf{u}} \in\left[L^{2}(\hat{T})\right]^{2}$ by

$$
P_{T} \hat{\mathbf{u}}(x, y)=\frac{1}{\left|\operatorname{det} J_{T}\right|} J_{T} \hat{\mathbf{u}}\left(\pi_{T}(r, s)\right),
$$

where $J_{T}$ denotes the Jacobian matrix of $\pi_{T}$.

Lemma 1 (Properties of Piola transformation, see e.g. [3]) For any $\mathbf{u} \in H(\operatorname{div}, T)$ and $v \in H^{1}(T)$ we have

$$
\begin{aligned}
\int_{T}(\nabla \cdot \mathbf{u}) v & =\int_{\hat{T}}(\hat{\nabla} \cdot \hat{\mathbf{u}}) \hat{v} ; \\
\int_{T} \mathbf{u} \cdot \nabla v & =\int_{\hat{T}} \hat{\mathbf{u}} \cdot \hat{\nabla} \hat{v} ; \\
\int_{\partial T} \mathbf{u} \cdot \mathbf{n}_{T} v & =\int_{\partial \hat{T}} \hat{\mathbf{u}} \cdot \hat{\mathbf{n}}_{\hat{T}} \hat{v},
\end{aligned}
$$

where $\hat{\mathbf{u}}=P_{T}^{-1} \mathbf{u}$ and $\hat{v}=\pi_{T}^{-1} v$.

Now we are ready to formulate the theorem that provides a construction of the basis.

Theorem 1 In the local Raviart-Thomas space $\mathbb{R} \mathbb{T}^{k}(T), k \in \mathbb{N}_{0}, T \in \mathscr{T}_{h}, T=$ $\left\{\mathbf{v}^{1}, \mathbf{v}^{2}, \mathbf{v}^{3}\right\}$ there exists a basis $\left\{\Phi_{i, l}^{\partial T}, \Psi_{m}^{T}\right\}, i=1,2,3, l=1, \ldots, k+1, m=$ $1, \ldots, 2 M, M=\frac{k(k+1)}{2}$ such that 
(B1)

$$
\left.\Phi_{i, l}^{\partial T} \cdot \mathbf{n}_{i^{\prime}}^{T}\right|_{i^{\prime}}=\delta_{i, i^{\prime}} L_{l}^{i}, \quad i, i^{\prime} \in\{1,2,3\}, \quad l \in\{1, \ldots, k+1\},
$$

where $E_{i}$ denotes the triangle's edge opposite to the vertex $\mathbf{v}^{i}, \mathbf{n}_{i}^{T}$ is the unit normal to the edge $E_{i}$ external to $T$ and $\left\{\mathbb{L}_{l}^{i}\right\}_{l=0}^{k}$ is orthonormal system of Legendre polynomials in $L^{2}\left(E_{i}\right)$.

(B2) $\Psi_{m}^{T}, m=1, \ldots, 2 M$ form basis in $L^{2}\left(\left[\mathbb{P}_{k-1}(\hat{T})\right]^{2}\right)$ and

$$
\left.\Psi_{m}^{T} \cdot \mathbf{n}_{i}\right|_{E_{i}}=0, \quad i \in\{1,2,3\}, m \in\{1, \ldots, 2 M\} ;
$$

Proof. Following [16] let us consider in the master element $\hat{T}$ vector functions

$$
\begin{gathered}
\mathbf{e}_{1}(r, s)=\frac{1}{2}\left(\begin{array}{c}
r+1 \\
s+1
\end{array}\right), \quad \mathbf{e}_{2}(r, s)=\frac{1}{2}\left(\begin{array}{c}
r-1 \\
s+1
\end{array}\right), \quad \mathbf{e}_{3}(r, s)=\frac{1}{2}\left(\begin{array}{c}
r+1 \\
s-1
\end{array}\right), \\
\mathbf{t}_{1}(r, s)=\frac{s+1}{2}\left(\begin{array}{c}
r+1 \\
s-1
\end{array}\right), \quad \mathbf{t}_{2}(r, s)=\frac{r+1}{2}\left(\begin{array}{c}
r-1 \\
s+1
\end{array}\right) .
\end{gathered}
$$

We define

$\hat{\Phi}_{i, l}(r, s)=\mathbb{L}_{l-1}(s) \mathbf{e}_{i}(r, s), i=1,2, \quad \hat{\Phi}_{3 l}(r, s)=\mathbb{L}_{l-1}(r) \mathbf{e}_{3}(r, s), l \in\{1, \ldots, k+1\}$, where $\left\{\mathbb{L}_{n}\right\}_{n=0}^{k}$ are (normalized) Legendre polynomials that form an orthonormal system in $L^{2}([-1,1])$. Since $\hat{\Phi}_{i, l}$ satisfies the property

$$
\left.\mathbf{e}_{1} \cdot \mathbf{n}_{j}\right|_{\hat{E}_{j}}=\frac{\sqrt{2}}{2} \delta_{1 j},\left.\quad \mathbf{e}_{i} \cdot \mathbf{n}_{j}\right|_{\hat{E}_{j}}=\delta_{i j}, \quad i \in\{2,3\}, j \in\{1,2,3\}
$$

we obtain

$$
\left.\hat{\Phi}_{i, l^{\prime}}^{\partial \hat{T}} \cdot \mathbf{n}_{i^{\prime}}^{\hat{T}}\right|_{i^{\prime}}=\delta_{i, i^{\prime}} \mathbb{L}_{l}^{i}
$$

Note that

$$
\int_{\hat{E}_{i}}\left(\hat{\Phi}_{i, l^{\prime}}^{\partial \hat{T}} \cdot \hat{\mathbf{n}}_{i}\right)\left(\hat{\Phi}_{i, l}^{\partial \hat{T}} \cdot \hat{\mathbf{n}}_{i}\right)=\delta_{l l^{\prime}}, \quad l, l^{\prime} \in\{1, \ldots, k+1\}, i \in\{1,2,3\} .
$$

Next we define $\hat{\Psi}_{m}=\hat{p}_{m}(r, s) \mathbf{t}_{1}(r, s), m=1, \ldots, M$ and $\hat{\Psi}_{m}=\hat{p}_{m-M}(r, s) \mathbf{t}_{2}(r, s)$, $m=M+1, \ldots, 2 M$, where polynomials $\hat{p}_{m}$ form the orthonormal Dubiner basis in $\mathbb{P}_{k-1}(\hat{T})$, cf. [10]. Since

$$
\left.\mathbf{t}_{j}(r, s) \cdot \mathbf{n}_{i}\right|_{\hat{E}_{i}}=0, j \in\{1,2\}, i \in\{1,2,3\}
$$

we have $\left.\hat{\Psi}_{m} \cdot \mathbf{n}_{i}\right|_{\hat{E}_{i}}=0$. Using the Piola transformation the respective basis in $\mathbb{R} \mathbb{T}^{k}(T), T \in \mathscr{T}_{h}$ is defined as

$$
\Phi_{i, l}^{\partial T}=P \circ \Phi_{i l} \circ \pi_{T}^{-1}, \Psi_{m}^{T}=P \circ \hat{\Psi}_{m} \circ \pi_{T}^{-1}
$$


and the required properties (B1) - (B2) follow directly from Lemma 1.

Next we will demonstrate how to recuperate the coefficients in respect to this basis from the degrees of freedom of a finite element in $\mathbb{R T}^{k}$ space.

Let us consider in $\left[\mathbb{P}_{k-1}(T)\right]^{2}$ a basis $\left\{\mathbf{P}_{m}^{T}\right\}_{m=1}^{2 M}$,

$$
\mathbf{P}_{m}^{T}=\left(\begin{array}{c}
p_{m} \\
0
\end{array}\right), m=1, \ldots, M ; \quad \mathbf{P}_{m}^{T}=\left(\begin{array}{c}
0 \\
p_{M-m}
\end{array}\right) j=M+1, \ldots, 2 M ;
$$

where polynomials $p_{m}$ form the Dubiner basis in $\mathbb{P}_{k-1}(T)$.

Lemma 2 Assume that for $\mathbf{u}_{h} \in \mathbb{R} \mathbb{T}^{k}\left(\mathscr{T}_{h}\right)$

$$
\mathbf{u}_{T}=\left.\mathbf{u}_{h}\right|_{T}=\sum_{i^{\prime}, l^{\prime}} c_{i^{\prime}, l^{\prime}}^{\partial T} \Phi_{i^{\prime}, l^{\prime}}^{\partial T}+\sum_{m^{\prime}} c_{m^{\prime}}^{T} \Psi_{m^{\prime}}^{T}
$$

be local representation in respect to the basis $\left\{\Phi_{i, l}^{\partial T}, \Psi_{m}^{T}\right\}$ in $\mathbb{R}^{k}(T)$.

Let

$$
\begin{gathered}
\mu_{i, l}^{\partial T}\left(\mathbf{u}_{T}\right)=\int_{E_{i}}\left(\mathbf{u}_{T} \cdot \mathbf{n}_{i}\right) \mathbb{L}_{l}^{i}, \quad i \in\{1,2,3\}, l \in 1, \ldots, k+1 ; \\
\mu_{m}^{T}\left(\mathbf{u}_{T}\right)=\int_{T} \mathbf{u}_{T} \cdot \mathbf{P}_{m}, \quad m \in\{1, \ldots, 2 M\}
\end{gathered}
$$

be the degrees of freedom of $\mathbf{u}_{T}$. Then

$$
c_{i, l}^{\partial T}=\mu_{i, l}^{\partial T}\left(\mathbf{u}_{T}\right), i \in\{1,2,3\}, l \in 1, \ldots, k+1 \text { and } \quad \mathbf{c}^{T}=G_{T}^{-1} \mathbf{F}^{T},
$$

where $\mathbf{c}^{T}=\left(c_{1}^{T}, \ldots, c_{2 M}^{T}\right)^{\prime}$

$$
G_{T}=\left[\int_{T} \Psi_{i}^{T} \cdot \mathbf{P}_{j}^{T}\right]_{2 M \times 2 M}, \quad \mathbf{F}_{T}=\left[\mu_{m}^{T}(\mathbf{u})-\sum_{i, l} \mu_{i, l}^{\partial T} \int_{T} \Phi_{i, l}^{\partial T} \cdot \mathbf{P}_{m}^{T}\right]_{1 \times 2 M} .
$$

Proof. From the edge moments (11) of (10) we have:

$$
\begin{gathered}
\mu_{i, l}^{\partial T}\left(\mathbf{u}_{T}\right)=\sum_{i^{\prime}, l^{\prime}} c_{i^{\prime}, l^{\prime}}^{\partial T} \int_{E_{i}}\left(\Phi_{i^{\prime}, l^{\prime}}^{\partial T} \cdot \mathbf{n}_{i}\right) \mathbb{L}_{l}^{i}+\sum_{m^{\prime}} c_{m^{\prime}}^{T} \int_{E_{i}}\left(\Psi_{m^{\prime}}^{T} \cdot \mathbf{n}_{i}\right) \mathbb{L}_{l}^{i} \\
=c_{i, l^{\prime}}^{\partial T} \int_{E} \mathbb{L}_{l^{\prime}} \mathbb{L}_{l}=c_{i, l}^{\partial T}
\end{gathered}
$$

owing to (B1), (B2) and orthogonality of the Legendre polynomials (9). Similarly from the element's moments (12) we obtain:

$$
\mu_{m}^{T}\left(\mathbf{u}_{T}\right)=\sum_{i^{\prime}, l^{\prime}} c_{i^{\prime}, l^{\prime}}^{\partial T} \int_{T} \Phi_{i^{\prime}, l^{\prime}}^{\partial T} \cdot \mathbf{P}_{m}^{T}+\sum_{m^{\prime}} c_{m^{\prime}}^{T} \int_{T} \Psi_{m^{\prime}}^{T} \cdot \mathbf{P}_{m}^{T}
$$

that is $G_{T} \mathbf{c}^{T}=\mathbf{F}_{T}$. 
Note 1 Let us note that Lemma 2 provides extremely cheap method for the flux recuperation from the moments: in each element of the mesh we only need to solve a small linear system.

Note 2 Since $\int_{T} \Psi_{m^{\prime}}^{T} \cdot \Psi_{m}^{T}=\left|J_{T}\right| \int_{\hat{T}} P_{T} \Psi_{m^{\prime}}^{T} \cdot P_{T} \Psi_{m}^{T}=\int_{\hat{T}}\left(J_{T} J_{T}^{\prime}\right) /\left|J_{T}\right| \hat{\Psi}_{m^{\prime}} \cdot \hat{\Psi}_{m}^{T}$, we immediately obtain $c_{m}^{T}=\mu_{m}^{T}\left(\mathbf{u}_{T}\right)-\sum_{i, l} \mu_{i, l}^{\partial T}\left(\mathbf{u}_{T}\right) \int_{T} \Phi_{i, l}^{\partial T} \cdot \Psi_{m}^{T}, \quad m \in 1, \ldots, 2 M$ for triangles where $\left(J_{T} J_{T}^{\prime}\right) /\left|J_{T}\right|=I d$. This situation occurs for rectangular equilateral elements for example, so for such structured triangular meshes the flux reconstruction can be calculated directly from the moments and does not require a solution of the local systems.

\section{Equilibrated flux reconstruction for discontinuous Galerkin method}

Let us present an application of the computational basis introduced in previous section to equilibrated fluxes reconstruction from discrete gradient of discontinuous Galerkin approximation to a solution of elliptic boundary value problem.

We consider in $\Omega$ next model problem:

$$
\begin{array}{rlr}
-\nabla \cdot(D \nabla u) & =f \quad \text { in } \Omega, \\
u & =g & \text { on } \partial \Omega .
\end{array}
$$

Here the diffusion coefficient $D>0$ is supposed to be constant in $\Omega, f \in L^{2}(\Omega)$ and $g \in H^{3 / 2}(\partial \Omega)$.

For a shape regular family $\mathscr{T}_{h}$ of triangular meshes in $\Omega$ we introduce the (discontinuous) finite element spaces $\mathscr{V}_{h}^{k}$ as:

$$
\mathscr{V}_{h}^{k}:=\left\{v_{h} \in L^{2}(\Omega):\left.v_{h}\right|_{T} \in \mathbb{P}_{k}(T), \forall T \in \mathscr{T}_{h}\right\} .
$$

Symmetric version of the interior penalty $\mathrm{dG}$ method is formulated as: find $u_{h} \in \mathscr{V}_{h}^{k}$ such that

$$
B_{h}\left(u_{h}, v_{h}\right)=F\left(v_{h}\right), \forall v_{h} \in \mathscr{V}_{h}^{k}
$$

where

$$
\begin{aligned}
B_{h}\left(u_{h}, v_{h}\right) & \left.=\int_{\mathscr{T}_{h}} D \nabla_{h} u_{h} \cdot \nabla_{h} v_{h}-\int_{\mathscr{E}}\left\{\left\{\mathbf{n}_{\mathscr{E}} \cdot D \nabla_{h} u_{h}\right\}\right\}\left[v_{h}\right]\right] \\
& \left.+\int_{\mathscr{E}}\left(-\left\{\left\{\mathbf{n}_{\mathscr{E}} \cdot D \nabla_{h} v_{h}\right\}\right\}+\gamma_{\mathscr{E}}\left[\left[v_{h}\right]\right]\right)\left[u_{h}\right]\right], \\
F(v) & =\int_{\Omega} f v+\int_{\mathscr{E}^{\partial}}\left(-\left\{\left\{\mathbf{n} \cdot D \nabla_{h} v_{h}\right\}\right\}+\gamma_{\mathscr{E}}\left[\left[v_{h}\right]\right]\right) g .
\end{aligned}
$$


Here we are using standard definition (see e.g. [9]) for discrete gradient, mean value and jump at the edges; the penalty parameter $\left.\gamma_{\mathscr{E}}\right|_{E}=2.5 D(k+1)^{2} h_{E}^{-1}$ is considered to be reasonable ([17]) for the stabilization.

For $v \in H^{1}\left(\mathscr{T}_{h}\right)+\mathscr{V}_{h}^{k}$ we define the energy norm associated with the dG method by

$$
\left\|v_{h}\right\|_{d G}=\left(\left\|D^{\frac{1}{2}} \nabla_{h} v_{h}\right\|_{L^{2}(\Omega)}^{2}+\int_{\mathscr{E}} \gamma_{\mathscr{E}}\left[\left[v_{h}\right]^{2}\right)^{\frac{1}{2}} .\right.
$$

For the energy norm of the error in $\mathrm{dG}$ approximation the following a priori estimate holds true, see e.g.[18].

Theorem 2 Let $u \in H^{k+1}(\Omega)$ be a weak solution to (14) and $u_{h} \in \mathscr{V}_{h}^{k}$ be the $d G$ finite element approximation of $u$. Then the estimate

$$
\left\|u-u_{h}\right\|_{d G} \leq C h^{k}\|u\|_{H^{k+1}(\Omega)}
$$

holds with a constant $C>0$ independent of $h$.

We will term the potential the weak solution $u \in H^{1}(\Omega)$ to the problem (14) and the flux $\sigma(u)=-D \nabla u \in H(\operatorname{div}, \Omega)$. Similarly, the $\mathrm{dG}$ solution $u_{h} \in \mathscr{V}_{h}^{k}$ to (15) is said to be discrete $\mathrm{dG}$ potential and the discrete $\mathrm{dG}$ flux is defined as $\sigma_{h}(u)=-D \nabla_{h} u_{h}$.

Definition 1. A vector field $\mathbf{s}_{h} \in H(\operatorname{div}, \Omega)$ is called equilibrated up to order $l \in \mathbb{N}_{0}$ if $\operatorname{div} \mathbf{s}_{h}-\pi_{h}^{l}(f)=0$, where $\pi_{h}^{l}: L^{2}(\Omega) \rightarrow \mathscr{V}_{h}^{l}$ denotes the orthogonal projection operator.

Following [13], let us consider a flux $\mathbf{t}_{h}^{k-1}\left(u_{h}\right) \in \mathbb{R}^{k-1}\left(\mathscr{T}_{h}\right)$ with the degrees of freedom of (11) and (12) locally prescribed by :

$$
\begin{aligned}
k \geq 1: & \mu_{i, l}^{\partial T}\left(\mathbf{t}_{h}^{k-1}\left(u_{h}\right)\right)=\int_{E_{i}}\left(-\left\{\left\{\mathbf{n} \cdot D \nabla_{h} u_{h}\right\}\right\}+\gamma_{E}\left[\left[u_{h}\right]\right]_{g}\right) \mathbb{L}_{l}^{i} \\
& E_{i} \in \partial T, i \in\{1,2,3\}, l \in 1, \ldots, k \\
k \geq 2: & \mu_{m}^{T}\left(\mathbf{t}_{h}^{k-1}\left(u_{h}\right)\right)=-\int_{T} D \nabla_{h} u_{h} \cdot \mathbf{P}_{m} \\
+ & \sum_{i} \int_{E_{i}} \chi_{\mathrm{e}}\left(E_{i}\right) D\left(\mathbf{P}_{m} \cdot \mathbf{n}_{i}\right)\left[\left[u_{h}\right]_{g}\right. \\
& m=1, \ldots, 2 M
\end{aligned}
$$

where

$$
\left[\left[u_{h}\right]\right]_{g}=\left\{\begin{array}{c}
{\left[\left.\left[u_{h}\right]\right|_{E}, \text { for } E \in \mathscr{E}^{\mathrm{i}},\right.} \\
\left.u_{h}\right|_{E}-g, \text { for } E \in \mathscr{E}^{\mathrm{D}} .
\end{array}\right.
$$

Note that this definition is correct since the numerical fluxes of $\mathrm{dG}$ method are uniquely defined at the edges of the mesh. Moreover, we will refer to the following lemma.

Lemma 3 Let $u$ be a weak solution to (14) and $u_{h} \in \mathscr{V}_{h}^{k}$ be the $d G$ finite element approximation of $u$. The flux $\mathbf{t}_{h}^{k-1}\left(u_{h}\right)$, defined by (18) - (19), is equilibrated up to 
order $k-1$ and there exists a constant $C>0$, independent of $h$, such that

$$
\left\|D^{\frac{1}{2}} \nabla u-D^{-\frac{1}{2}} \mathbf{t}_{h}^{k-1}\left(u_{h}\right)\right\|_{L^{2}(\Omega)} \leq C\left\|u-u_{h}\right\|_{d G} .
$$

See [13] for the proof and more details.

For computational reconstruction of $\mathbf{t}_{h}^{k-1}\left(u_{h}\right)$ from the prescribed moments (18) and (19) we use the algorithm presented in Lemma 2.

\section{Numerical examples}

To demonstrate a potential of the suggested algorithm we consider an application to adaptive mesh refinement in $\mathrm{dG}$ approximation of elliptic boundary value problems, where the equilibrated fluxes are used in a posteriori error estimator in the energy norm (16).

We consider the error estimator introduced in [15]

$$
\eta^{2}\left(u_{h}\right)=\sum_{T \in \mathscr{T}_{h}} \eta^{2}(T)=\sum_{T \in \mathscr{T}_{h}}\left(\left(\eta_{\mathscr{O}}(T)+\eta_{\nabla}(T)\right)^{2}+\eta_{\mathscr{H}}^{2}(T)\right)
$$

where $\eta_{\mathscr{O}}(T)=\left\|f-\nabla \cdot \mathbf{t}_{h}\left(u_{h}\right)\right\|_{L^{2}(T)}$ is the oscillations term, $\eta_{\nabla}(T)=\| D^{-\frac{1}{2}}\left(\sigma_{h}\left(u_{h}\right)-\right.$ $\left.\mathbf{t}_{h}\left(u_{h}\right)\right) \|_{L^{2}(T)}$ measures the deviation of the discrete flux $\sigma_{h}\left(u_{h}\right)$ from $\mathbf{H}(\operatorname{div}, \Omega)$ and $\eta_{\mathscr{H}}(T)=\left\|D^{\frac{1}{2}}\left(\nabla_{h}\left(u_{h}\right)-\nabla_{h}\left(u_{h}^{O}\right)\right)\right\|_{L^{2}(T)}$ measures the deviation of $u_{h}$ from $H^{1}(\Omega)$. Here $u_{h}^{O} \in H^{1}(\Omega)$ is Oswald interpolator of $u_{h}$ and the equilibrated flux $\mathbf{t}_{h}^{k-1}\left(u_{h}\right)$ is reconstructed by prescription from (18) - (19) in the computational basis $\left\{\Phi_{i, l}^{\partial T}, \Psi_{m}^{T}\right\}$ in $\mathbb{R} \mathbb{T}^{k}(T)$. This type of estimator has proven to be reliable, efficient and robust with respect to polynomial order of approximation space, see [4],[15]. The quality of the error estimator $\eta$ is assessed in terms of the effectivity index $\mathscr{I}_{\eta}=\frac{\eta\left(u_{h}\right)}{\left\|D^{\frac{1}{2}}\left(\nabla u-\nabla_{h} u_{h}\right)\right\|_{L^{2}(\Omega)}}$ evaluated on sequences of uniformly and adaptively refined meshes.

\subsection{Test case 1: uniform mesh refinement}

Firstly we confirm the order of approximation of the exact flux by the reconstructed flux for a smooth solution to elliptic problem. So let us consider the model problem (14) in $\Omega=(0,1)^{2}$ with $D=1$, the right-hand side and the homogeneous Dirichlet boundary condition corresponding to the exact solution $u(x, y)=\sin (\pi x) \sin (\pi y)$. The model problem was solved numerically using the $\mathrm{dG}$ method of order $k=$ $1,2,3,4$ on a sequence of meshes obtained by successive uniform bisection from the initial unstructured mesh of 48 elements. 
Let us denote by $e\left(u_{h}\right)=\left\|D^{-\frac{1}{2}}\left(\sigma(u)-\sigma_{h}\left(u_{h}\right)\right)\right\|_{L^{2}(\Omega)}$ the error in dG approximation, by $e\left(\mathbf{t}_{h}\right)=\left\|D^{-\frac{1}{2}}\left(\sigma(u)-\mathbf{t}_{h}\left(u_{h}\right)\right)\right\|_{L^{2}(\Omega}$ the error in approximation of the exact flux by the reconstructed Raviart-Thomas flux and by $e\left(\nabla \cdot \mathbf{t}_{h}\right)=\| f-\nabla$. $\mathbf{t}_{h}\left(u_{h}\right) \|_{L^{2}(\Omega)}$ the error in equilibration of the reconstructed flux. In Table 1 we show the errors and the convergence rates of the $\mathrm{dG}$ method and of the equilibrated flux, reconstructed from discrete solution using suggested computational basis. We observe that the $\mathrm{dG}$ method accurately approximate the exact solution to the problem and exhibits the optimal order of convergence $k$ predicted in the Theorem 2. The error in approximation of the exact flux by reconstructed equilibrated flux is also of order $k$, which is the optimal order of approximation of the exact flux by dG method and the optimal order of the projection of the exact flux on the Raviart-Thomas space $\mathbb{R} \mathbb{T}^{k-1}$. Finally, the order of convergence of $\nabla \cdot \mathbf{t}_{h} \rightarrow f$ is $k+1$, that is the flux $\mathbf{t}_{h}$ is equilibrated up to order $k-1$.

Table 1 Convergence rates $o\left(e\left(u_{h}\right)\right)$ and $o\left(\mathbf{t}_{h}\left(u_{h}\right)\right)$ for different orders of approximation calculated for the refinement of order $N_{r}$.

\begin{tabular}{|c|c|c|c|c|c|c|c|}
\hline$k$ & $N_{r}$ & $e\left(u_{h}\right)$ & $o\left(e\left(u_{h}\right)\right)$ & $e\left(\mathbf{t}_{h}\right)$ & $o\left(e\left(\mathbf{t}_{h}\right)\right)$ & $e\left(\nabla \cdot \mathbf{t}_{h}\right)$ & $o\left(e\left(\nabla \cdot \mathbf{t}_{h}\right)\right)$ \\
\hline \multirow{4}{*}{1} & 1 & $2.779 \times 10^{-1}$ & 2.951 & $3.664 \times 10^{-1}$ & 2.605 & $6.013 \times 10^{-2}$ & 5.544 \\
& 2 & $1.394 \times 10^{-1}$ & 0.995 & $1.879 \times 10^{-1}$ & 0.964 & $1.521 \times 10^{-2}$ & 1.983 \\
& 3 & $6.984 \times 10^{-2}$ & 0.997 & $9.425 \times 10^{-2}$ & 0.995 & $3.813 \times 10^{-3}$ & 1.996 \\
& 4 & $3.495 \times 10^{-2}$ & 0.999 & $4.711 \times 10^{-2}$ & 1.000 & $9.540 \times 10^{-4}$ & 1.999 \\
& 5 & $1.749 \times 10^{-2}$ & 0.999 & $2.354 \times 10^{-2}$ & 1.001 & $2.385 \times 10^{-4}$ & 2.000 \\
\hline \multirow{4}{*}{2} & 1 & $2.480 \times 10^{-2}$ & 5.058 & $3.184 \times 10^{-2}$ & 4.275 & $3.725 \times 10^{-3}$ & 8.005 \\
& 2 & $6.227 \times 10^{-3}$ & 1.994 & $7.819 \times 10^{-3}$ & 2.026 & $4.685 \times 10^{-4}$ & 2.991 \\
& 3 & $1.561 \times 10^{-3}$ & 1.996 & $1.944 \times 10^{-3}$ & 2.008 & $5.864 \times 10^{-5}$ & 2.998 \\
& 4 & $3.910 \times 10^{-4}$ & 1.998 & $4.852 \times 10^{-4}$ & 2.003 & $7.332 \times 10^{-6}$ & 3.000 \\
& 5 & $9.783 \times 10^{-5}$ & 1.999 & $1.212 \times 10^{-4}$ & 2.001 & $9.165 \times 10^{-7}$ & 3.000 \\
\hline \multirow{4}{*}{3} & 1 & $1.011 \times 10^{-3}$ & 7.457 & $1.294 \times 10^{-3}$ & 6.806 & $2.743 \times 10^{-4}$ & 9.262 \\
& 2 & $1.294 \times 10^{-4}$ & 2.965 & $1.648 \times 10^{-4}$ & 2.973 & $1.697 \times 10^{-5}$ & 4.015 \\
& 3 & $1.623 \times 10^{-5}$ & 2.995 & $2.050 \times 10^{-5}$ & 3.007 & $1.059 \times 10^{-6}$ & 4.003 \\
& 4 & $2.029 \times 10^{-6}$ & 3.000 & $2.547 \times 10^{-6}$ & 3.008 & $6.613 \times 10^{-8}$ & 4.001 \\
& 5 & $2.536 \times 10^{-7}$ & 3.001 & $3.172 \times 10^{-7}$ & 3.005 & $4.133 \times 10^{-9}$ & 4.000 \\
\hline & 1 & $6.619 \times 10^{-5}$ & 8.177 & $8.910 \times 10^{-5}$ & 7.072 & $8.448 \times 10^{-6}$ & 11.581 \\
4 & 2 & $4.042 \times 10^{-6}$ & 4.033 & $5.414 \times 10^{-6}$ & 4.041 & $2.801 \times 10^{-7}$ & 4.915 \\
& 3 & $2.514 \times 10^{-7}$ & 4.007 & $3.371 \times 10^{-7}$ & 4.005 & $8.856 \times 10^{-9}$ & 4.983 \\
& 4 & $1.570 \times 10^{-8}$ & 4.001 & $2.109 \times 10^{-8}$ & 3.999 & $2.775 \times 10^{-10}$ & 4.996 \\
& 5 & $9.812 \times 10^{-10}$ & 4.000 & $1.319 \times 10^{-9}$ & 3.999 & $1.161 \times 10^{-11}$ & 4.579 \\
\hline
\end{tabular}

\subsection{Test case 2: adaptive mesh refinement}

Inspired by propagation of saturation front in a two-fase flow in porous media, we consider here the model problem (14) in $\Omega=(0,1)^{2}$ with the solution 
$u(x, y)=x(x-1) y(y-1) \arctan \left(60 \sqrt{\left.(x-5 / 4)^{2}+(y+1 / 4)^{2}\right)-1}\right)$, that exhibits a steep front in the interior of the domain. This example is commonly used for testing adaptive refinement algorithms for elliptic equations, c.f. [21]. Here we also consider $D=1$, and the right-hand side and the homogeneous Dirichlet boundary condition correspond to the exact solution.

Meshes are adapted from the same initial mesh, using a refinement strategy based on the method proposed by Dörfler, whereby the elements in a minimal set $\mathscr{M} \subset \mathscr{T}_{h}$, such that $\sum_{T \in \mathscr{M}} \eta(T) \geq \theta \sum_{T \in \mathscr{T}_{h}} \eta(T)$, are refined. Elements are refined using the longest edge bisection technique and additional refinements of the mesh are considered in order to eliminate hanging nodes.

Figure 1 displays in the first column the energy norm of the error and the convergence order calculated for uniform and adaptive mesh refinements with $\theta=0.25$, $\theta=0.5$ and $\theta=0.75$ in the Dörfler marking as a function of DOF on a logarithmic scale for order of $\mathrm{dG}$ method $k=1$ to $k=4$. The second column shows the respective effectivity indices and the third column presents the adaptively refined meshes corresponding to the error $\approx 0.01$ for $\theta=0.25$. We can see that for given order of $\mathrm{dG}$ method the energy norm of the errors are very close for all values of the parameter in the Dörfler marking and asymptotically exhibit the optimal convergence rates. The effectivity indices remain above 1 and are asymptotically close to 1 even the order of approximation increases. We observe also that the number of DOF necessary to achieve the same global approximation error decreases with increasing polynomial degree $k$.

\section{Conclusions}

A specific modal computational basis, in which the coordinates of the equilibrated fluxes can be easy calculated from the numerical fluxes of $\mathrm{dG}$ method, is designed for high order Raviart-Thomas space. Optimal convergence of equilibrated fluxes and the robustness of the reconstruction method in respect to the order of $\mathrm{dG}$ method are confirmed numerically. Adaptive mesh refinement, guided by the equilibrated error estimator calculated in this basis, exhibits robust effectivity index and provides final meshes with less DOF for the same error tolerance for higher orders of the dG method.

Acknowledgements The authors gratefully acknowledge the support by $\mathrm{CNPq}$, Brazil, grant 477935/2013-3.

\section{References}

1. R. A. Adams. Sobolev Spaces. Academic Press, 1975. 

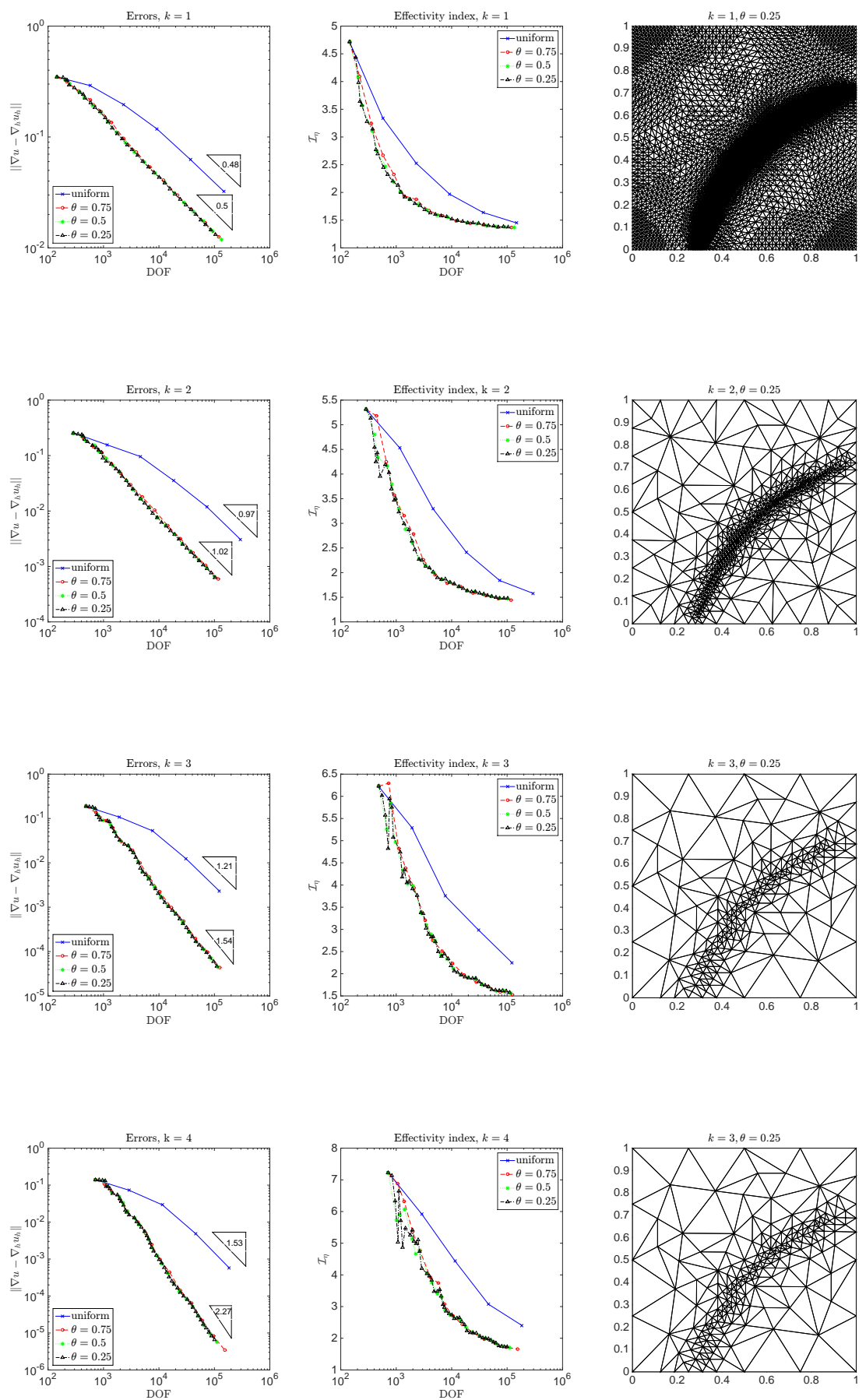

Fig. 1 The energy norm of error, the convergence order (left column) and the effectivity index (middle column) as a function of the degrees of freedom (DOF) on a logarithmic scale for various $\theta$ in the Dörfler marking and for various orders of $\mathrm{dG}$ method. Right column: adaptively refined meshes, corresponding to the error $\approx 0.01$ in the energy norm, with DOF number $N_{D O F}=104991$ for $k=1, N_{D O F}=7692$ for $k=2, N_{D O F}=4270$ for $k=3$ and $N_{D O F}=3930$ for $k=4$. 
2. C. Bahriawati and C. Carstensen. Three matlab implementations of the lowest-order RaviartThomas MFEM with a posteriori error control. Computational Methods in Applied Mathematics, 5(4):333-361, 2005.

3. D. Boffi, F. Brezzi, and M. Fortin. Mixed finite element methods and applications, volume 44 of Springer Series in Computational Mathematics. Springer, Heidelberg, 2013.

4. D. Braess, T. Fraunholz, and R. H. W. Hoppe. An equilibrated a posteriori error estimator for the interior penalty discontinuous Galerkin method. SIAM J. Numer. Anal., 52(4):2121-2136, 2014.

5. D. Braess, V. Pillwein, and J. Schöberl. Equilibrated residual error estimates are $p$-robust. Comput. Methods Appl. Mech. Engrg., 198(13-14):1189-1197, 2009.

6. S. C. Brenner and L. R. Scott. The Mathematical Theory of Finite Element Methods. Springer, 1994.

7. E. Creusé and S. Nicaise. A posteriori error estimator based on gradient recovery by averaging for discontinuous Galerkin methods. J. Comput. Appl. Math., 234(10):2903-2915, 2010.

8. E. Creusé and S. Nicaise. A posteriori error estimator based on gradient recovery by averaging for convection-diffusion-reaction problems approximated by discontinuous Galerkin methods. IMA J. Numer. Anal., 33(1):212-241, 2013.

9. D. A. Di Pietro and A. Ern. Mathematical Aspects of Discontinuous Galerkin Methods, volume 69 of Mathématiques \& Applications. Springer, 2011.

10. M. Dubiner. Spectral methods on triangles and other domains. J. Sci. Comput., 6(4):345-390, 1991.

11. A. Erm and I. Mozolevski. Discontinuous Galerkin method for two-component liquid gas porous media flows. Computational Geosciences, 16:677-690, 2012.

12. A. Ern, I. Mozolevski, and L. Schuh. Accurate velocity reconstruction for discontinuous Galerkin approximations of two-phase porous media flows. C. R. Math. Acad. Sci. Paris, 347(9-10):551-554, 2009.

13. A. Ern, S. Nicaise, and M. Vohralík. An accurate $\mathbf{H}($ div $)$ flux reconstruction for discontinuous Galerkin approximations of elliptic problems. C. R. Math. Acad. Sci. Paris, 345(12):709-712, 2007.

14. A. Ern and M. Vohralík. Flux reconstruction and a posteriori error estimation for discontinuous Galerkin methods on general nonmatching grids. C. R. Math. Acad. Sci. Paris, 347(7-8):441444, 2009.

15. A. Ern and M. Vohralík. Polynomial-degree-robust a posteriori estimates in a unified setting for conforming, nonconforming, discontinuous Galerkin, and mixed discretizations. SIAM J. Numer. Anal., 53(2):1058-1081, 2015.

16. V. Ervin. Computational bases for $R T_{k}$ and $B D M_{k}$ on triangles. Comput. Math. Appl., 64(8):2765-2774, 2012.

17. J. S. Hesthaven and T. Warburton. Nodal discontinuous Galerkin methods, volume 54 of Texts in Applied Mathematics. Springer, New York, 2008. Algorithms, analysis, and applications.

18. P. Houston, C. Schwab, and E. Süli. Discontinuous hp-finite element methods for advectiondiffusion problems. SIAM Journal of Numerical Analysis, 39(6):2133-2163, 2002.

19. K. Y. Kim. A posteriori error estimators for locally conservative methods of nonlinear elliptic problems. Appl. Numer. Math., 57(9):1065-1080, 2007.

20. J. M. Melenk and B. I. Wohlmuth. On residual-based a posteriori error estimation in $\mathrm{hp}$ FEM. Adv. Comput. Math., 15(1-4):311-331 (2002), 2001. A posteriori error estimation and adaptive computational methods.

21. W. F. Mitchell. A collection of 2D elliptic problems for testing adaptive grid refinement algorithms. Appl. Math. Comput., 220:350-364, 2013.

22. I. Mozolevski and S. Prudhomme. Goal-oriented error estimation based on equilibrated-flux reconstruction for finite element approximations of elliptic problems. Comput. Methods Appl. Mech. Engrg., 288:127-145, 2015. 